\title{
DIC imaging for identification of motor and sensory nerves
}

\author{
Dayu Chen*, Yuxiang $\mathrm{Wu}^{\dagger} \star$, Tao Sui ${ }^{\S}$, Shaoqun Zeng ${ }^{\dagger}$, \\ Xiaojian $\mathrm{Cao}^{\S, \boldsymbol{\uparrow}, * *}$ and Xiaohua $\mathrm{Lv}^{\dagger}, \|, * *$ \\ *Department of Neurosurgery \\ Wuhan General Hospital of Guangzhou Military Command of PLA \\ 627 Wuluo Road, Wuhan 430070, P. R.China \\ ${ }^{\dagger}$ Britton Chance Center for Biomedical Photonics \\ Wuhan National Laboratory for Optoelectronics \\ Huazhong University of Science and Technology \\ Wuhan 430074, P. R. China \\ $\$$ School of Physical Education, Jianghan University \\ Wuhan 430056, P. R. China \\ $\S_{\text {First Affiliated Hospital of Nanjing Medical University }}$ \\ Nanjing 210029, P. R. China \\ Ixiaojiancao@gmail.com \\ "Ixhlv@hust.edu.cn
}

Received 5 February 2016

Accepted 7 March 2016

Published 18 April 2016

\begin{abstract}
Identification of motor and sensory nerves is important in applications such as nerve injury repair. Conventional practice relies on time consuming staining methods for this purpose. Here, we use laser scanning infrared differential interference contrast (IR-DIC) microscopy for label-free observation of the two types of nerve. Ventral and dorsal nerve roots of adult beagle dogs were collected and sections of different thicknesses were imaged with an IR-DIC microscope. Different texture patterns of the IR-DIC images of the motor and sensory nerve can be distinguished when the section thickness increases to $40 \mu \mathrm{m}$. This suggests that nerve fibers in motor and sensory nerves have different distribution patterns. The result hints a potential new way for more rapid identification of nerve type in peripheral nerve repair surgery.
\end{abstract}

Keywords: Differential interference microscopy; nerve repair; nerve root; image pattern.

${ }^{* *}$ Corresponding authors.

This is an Open Access article published by World Scientific Publishing Company. It is distributed under the terms of the Creative Commons Attribution 4.0 (CC-BY) License. Further distribution of this work is permitted, provided the original work is properly cited. 


\section{Introduction}

Peripheral nerve system contains motor and sensory nerves, which are classified according to the type of the majority nerve fibers inside. For trauma patients with severe peripheral nerve injuries, nerve repair surgery may be required to reconnect divided nerve. To get the best outcome of the repair surgery, it is essential to discriminate motor and sensory nerve bundles and make correct connections between the distal and proximal part of the same type of injured nerve bundle. ${ }^{1,2}$

Various ways have been proposed to identify motor and sensory nerves. Sunderland examined the sequential slices of main nerves of human and drew the contour of fascicles inside to get a distribution map. ${ }^{3,4}$ But individual variation and trauma induced position change limit its application. Identification using electrical stimulation has also been applied. With microneurography technique, fine needles are inserted into peripheral nerves and patients are stimulated at sensory and motor nerve fibers and reported sensations. ${ }^{5}$ This method, however, requires the patients to remain conscious as the injuries are fresh. Staining based on different chemical compositions, such as acetylcholinesterase (AChE) histochemical staining, are applied. ${ }^{6-10}$ However, the procedure is time consuming due to the trace amount of the targeting constituents and the slow staining process.

With staining, the internal nerve fibers showed similar circular shapes in the images of thin sections of both motor and sensory nerves. By observing thicker sections of motor and sensory nerve samples with confocal fluorescence microscope, we showed that there exist different microstructural patterns of the internal fibers between motor and sensory nerves. ${ }^{11}$ To clearly see the nerve fibers inside, immunohistology staining or fluorescence labeling was employed.

Label-free microscopy is an important pursuit for imaging biological samples. ${ }^{12,13}$ As a classic label-free microscopy, differential interference contrast (DIC) microscopy is widely used to observe structure in unstained living cells and isolated organelles. ${ }^{14-16}$ Combined with infrared light source, the tissue penetration depth can be improved to facilitate imaging of thick or high scattering samples, such as living brain slices. ${ }^{17}$ In this paper, we attempted to use laser scanning infrared differential interference contrast (IR-DIC) microscopy for label-free observation of motor and sensory nerves. Ventral and dorsal spinal nerve roots of beagle dog, which are composed of pure motor and sensory nerve fibers, respectively, were used as the sample. Sections of the two types of nerves with different thicknesses were prepared and imaged. Texture patterns were analyzed, and the results showed that different texture patterns appear at thick nerve sections and may be used for differentiating the two types of nerves.

\section{Materials and Methods}

Twelve adult beagle dogs were anesthetized with sodium pentobarbital and then killed. From all the 12 of the dogs, canalis vertebralis were opened, and both anterior and posterior roots were harvested from L1-S2. 126 anterior roots and 126 posterior roots were collected. These nerves were kept in the liquid nitrogen and unfrozen at room temperature before section preparation. Spinal nerve roots were excised into pieces of 1-2 mm length and fixed in $4 \%$ paraformaldehyde for $24 \mathrm{~h}$ or $95 \%$ ethanol for $20 \mathrm{~min}$. After washing for three times in 0.1 M PBS containing (in mmol/L): $29 \mathrm{Na} 2 \mathrm{HPO} 4,2.96 \mathrm{NaH} 2 \mathrm{PO} 4$, and $8 \mathrm{NaCl}$, the dissected nerve was marinated in $25 \%$ saccharose solution for 15 min. Finally, transverse sections of different thicknesses were obtained from a cryostat microtome (CM1900, Leica) and coverslipped with glycerin. The animal experiments were performed following procedures approved by the institutional animal ethics committee of Nanjing Medical University and Huazhong University of Science and Technology.

A custom-made laser scanning IR-DIC microscope was used to image sections of spinal nerve roots. Briefly, a Ti: Sapphire laser (Ultra II, Coherent) was tuned to $800 \mathrm{~nm}$. The laser beam was collimated and coupled to a laser scanning microscope (BX61WI with FV300 confocal scanner, Olympus). Laser intensity was adjusted using a motorized ND filter wheel. The microscope was configured for DIC observation, a PMT is coupled to the transmission illumination part of the microscope to detect the transmitted laser. A $40 \mathrm{X}$ water immersion objective (LUMPLFLN 40XW, Olympus) was used. Images were acquired with fluoview software (Olympus). The system is shown in Fig. 1.

Texture analysis was performed for the acquired images to visualize the layout pattern of the internal nerve fibers. A neighborhood filtering was carried 


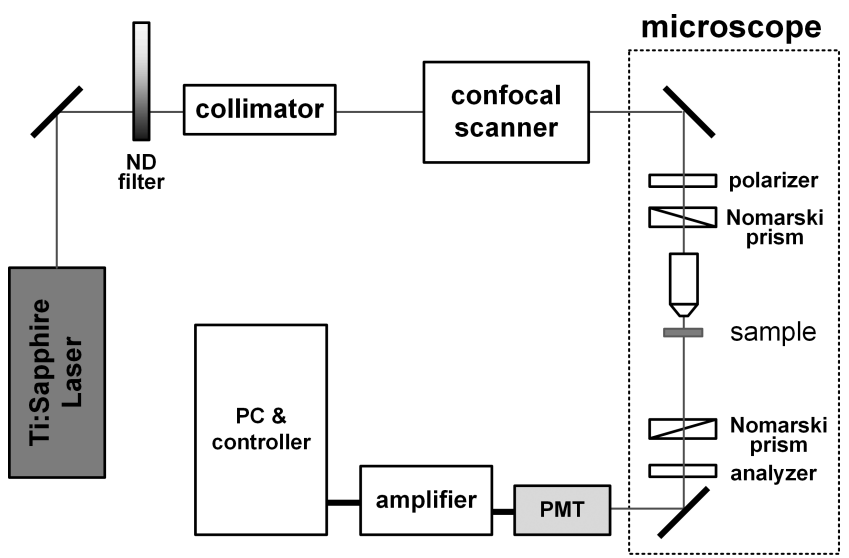

Fig. 1. System setup of the laser scanning infrared DIC microscope. An upright microscope with Nomarski prism based DIC optics is used. Laser passes through a ND filter and a collimator for adjusting the laser power and beam collimation. After that, the laser beam is coupled to a confocal scanner connected with the microscope. A PMT with amplifier module is connected to the transmission lamp port of the microscope. The amplified signal is connected to the confocal controller and the $\mathrm{PC}$ for image construction.

out, in which the local variance of each 3-by-3 neighborhood was calculated as the new value of the center pixel. With this method, the fiber boundary can be enhanced.

Individual nerve fibers can be identified in the DIC images. To give a quantified description, the classic Otsu's method, by maximizing the foreground and background variance, was used to determine the image threshold for converting the DIC images into binary images. Individual nerve fibers present as connected areas in the binary images. Two different shapes, stripe and circle, were roughly defined, according to the size of their occupied areas. The percentage of the summed areas of the two shapes in the images was calculated to characterize the DIC images of the ventral and dorsal nerve root sections with different section thicknesses.

\section{Results}

We imaged nerve sections of $10 \mu \mathrm{m}, 20 \mu \mathrm{m}$, and $40 \mu \mathrm{m}$ thick with the IR-DIC microscope. $Z$ stack imaging across the full thickness of the nerve sections was carried out and the maximum intensity projection images were shown in Fig. 2. As shown in Figs. 2(c)-2(f), at $10 \mu \mathrm{m}$ and $20 \mu \mathrm{m}$, there's no significant difference in the cross-section shape of the internal nerve fiber. However, at $40 \mu \mathrm{m}$ thick, the internal nerve fibers in the ventral nerve root present as stripe shapes, while those in the dorsal nerve root still look like circular shapes (Figs. 2(a) and $2(\mathrm{~b})$ ).

For images of $40 \mu \mathrm{m}$ thick sections, texture analysis was performed as described in the methods. As shown in Fig. 3, the boundary of the internal nerve fibers can be easily seen. In ventral nerve roots, most nerve fibers look like short stripes, some show as circular shapes (Figs. 1(a) and 1(b)). On the contrary, in dorsal nerve roots, most nerve fibers look like circular shapes. It can be deduced that the nerve fibers inside the ventral nerve root run in a

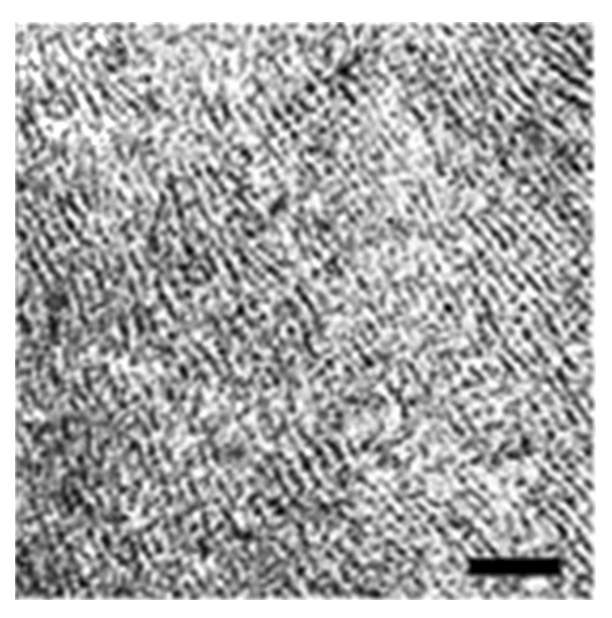

(a)

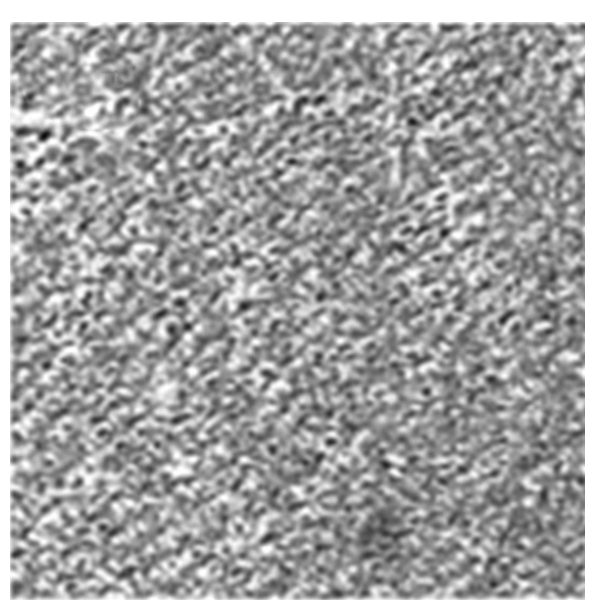

(b)

Fig. 2. IR-DIC images of nerve sections of different thicknesses. Images in the left column are results from ventral nerve root. Images in the right column are results from dorsal nerve root. (a) and (b) show the DIC images of $40 \mu \mathrm{m}$ cross-sections. (c) and (d) show the DIC images of $20 \mu \mathrm{m}$ cross-sections. (e) and (f) show the DIC images of $10 \mu \mathrm{m}$ cross-sections. Scale bar: $100 \mu \mathrm{m}$. 
D. Chen et al.

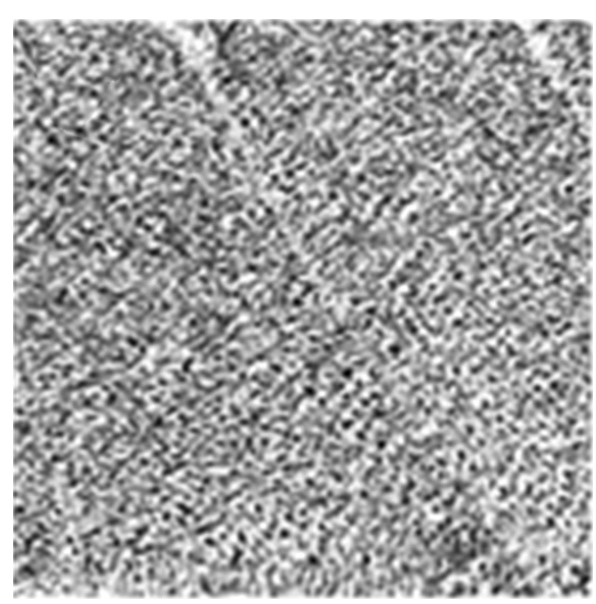

(c)

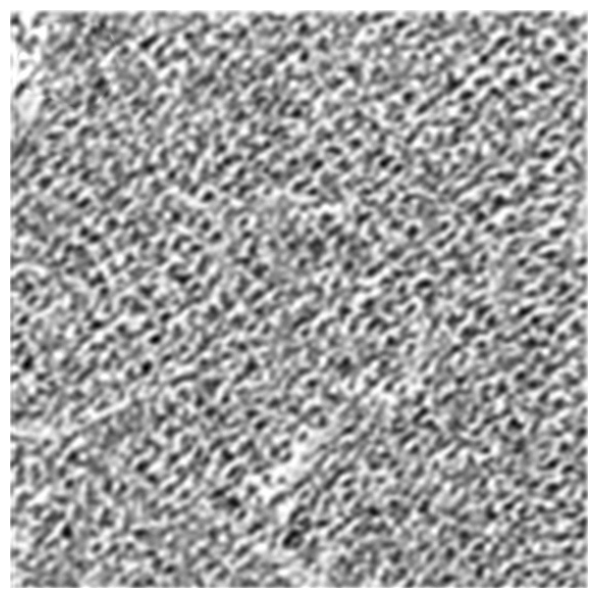

(e)

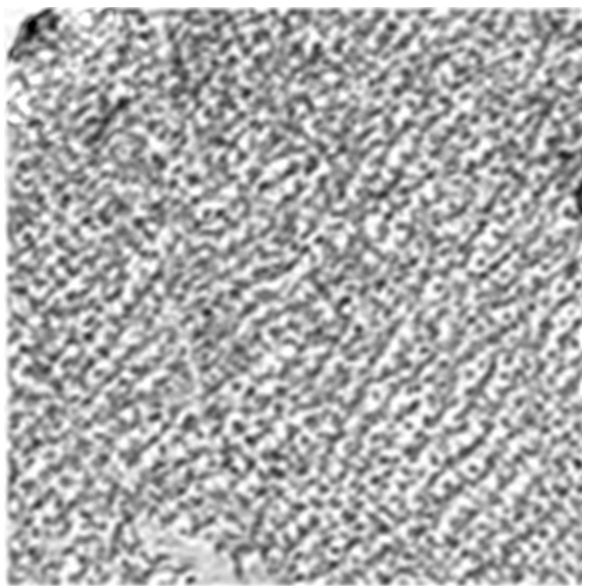

(d)

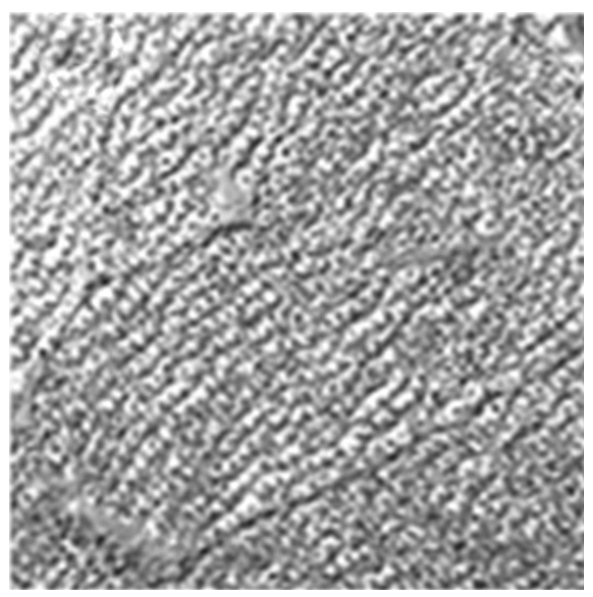

(f)

Fig. 2. (Continued)

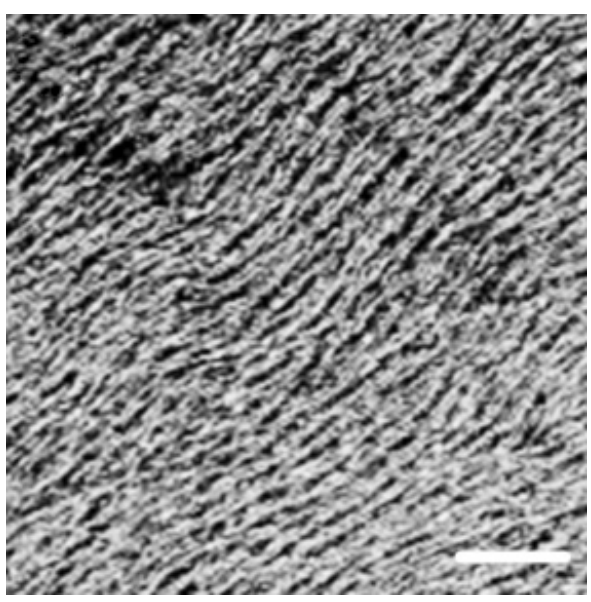

(a)

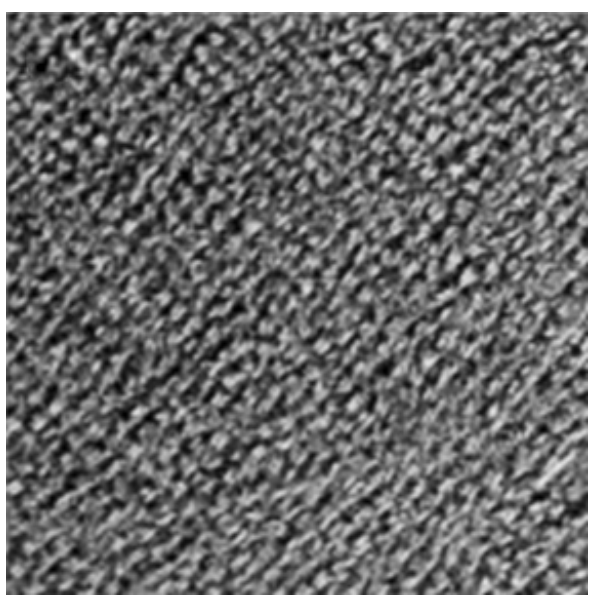

(b)

Fig. 3. IR-DIC images and texture filtered images from cross-sections of $40 \mu \mathrm{m}$ thick nerve samples. (a) and (b) are the DIC image of ventral and dorsal root, respectively. (c) and (d) are the corresponding texture filtered images from (a) and (b). Scale bar: $100 \mu \mathrm{m}$. 


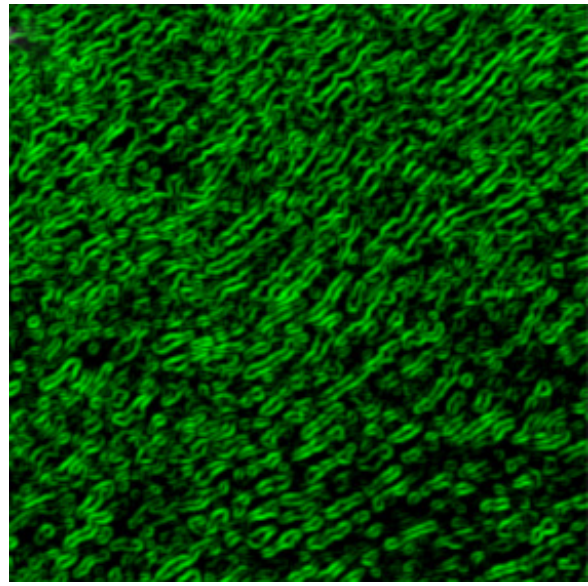

(c)

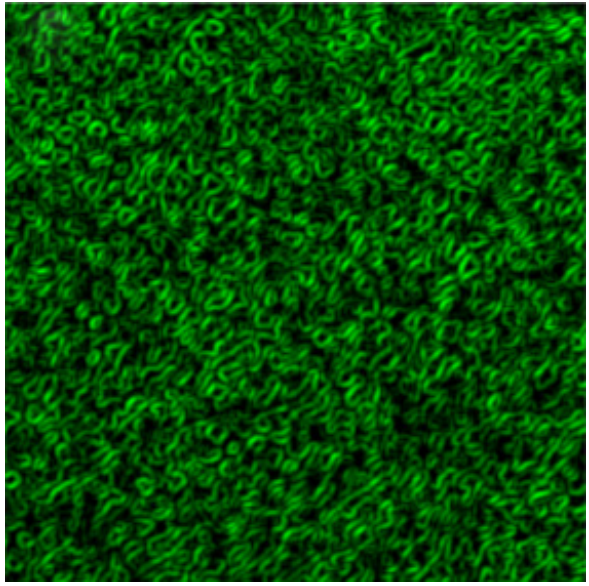

(d)

Fig. 3. (Continued)

more twisted way, so that the cross-section of the nerve fibers in a thicker nerve section present as short stripes. While, the nerve fibers in the dorsal nerve root run in parallel with the nerve root.

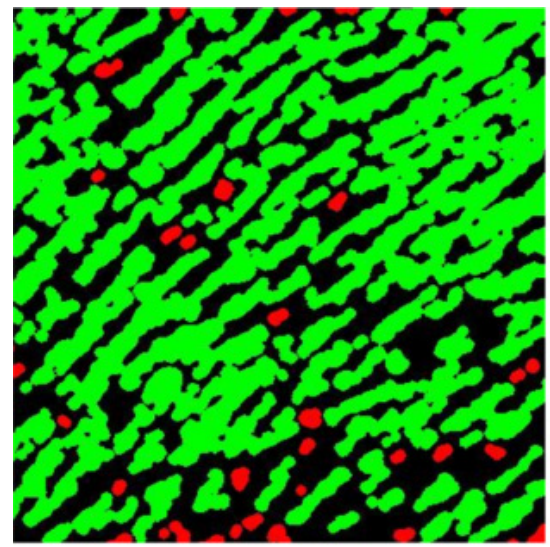

stripe area

(a)

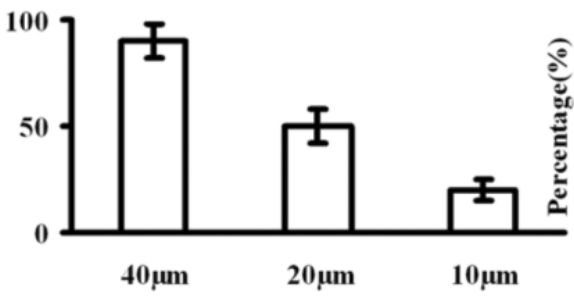

(c)
Figures 4(a) and 4(b) show color-coded binary images by thresholding the original DIC images of ventral and dorsal nerve roots, respectively. Nerve fibers present as connected areas of different shapes

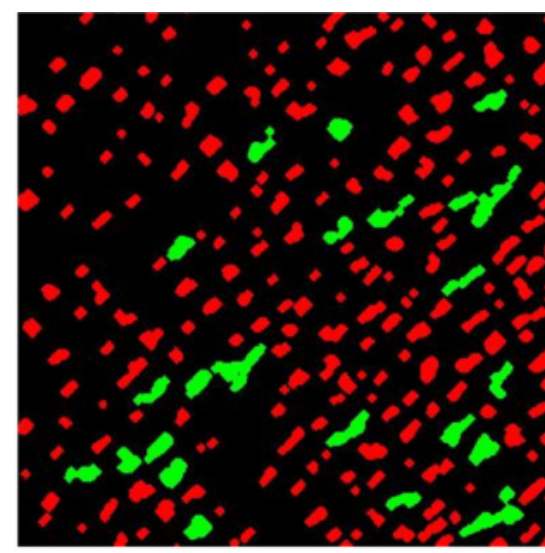

circular area

(b)

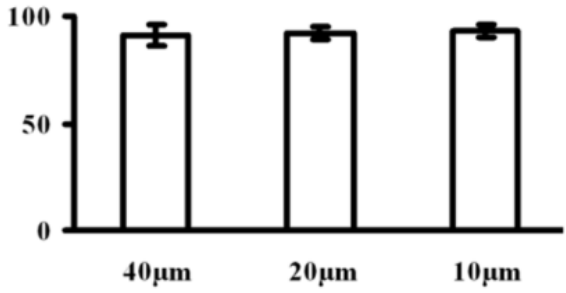

(d)

Fig. 4. Area percentage of stripe pattern in ventral nerve root and that of circular pattern in dorsal nerve root. Thresholding the DIC images and color coded the connected area into stripe area and circular area, according to their occupied areas. (a) and (b) show the results of ventral and dorsal nerve root of $40 \mu \mathrm{m}$ thick, respectively. (c) Percentage of stripe pattern in the DIC images of ventral nerve root with different section thicknesses. (d) Percentage of circular pattern in the DIC images of dorsal nerve root with different section thicknesses. 
in the binary images. The circular shape was roughly differentiated from the stripe shape by its smaller area, and the occupied areas were coded in red. The stripe areas were coded in green. We added up the occupied area of the circular shapes and that of the stripe shapes (stripe shapes tend to connect with the adjacent ones and their summed area can be directly counted) to calculate the percentage of the occupied area of the two shapes. The results were grouped according to the section thickness. As shown in Fig. 4(c), for the ventral nerve root, the percentage of stripe shape increases with nerve section thickness and reaches about $90 \%$ for nerve sections of $40 \mu \mathrm{m}$ thick. For the dorsal nerve root, the percentage of circular shape of the internal nerve fiber maintains at a level close to $90 \%$.

\section{Discussion and Conclusion}

In this paper, we show that with IR-DIC, the difference between ventral and dorsal nerve roots can be identified, using nerve sections of appropriate thickness. At $40 \mu \mathrm{m}$ section thickness, the DIC images of two spinal nerve roots presented two different patterns, which related to the distribution pattern of the internal nerve fibers. Therefore, it is possible to identify motor fibers from sensory fibers with this imaging method.

Previous work focus on the molecular composition between the motor and sensory nerves. In this way, staining is inevitable, which is time consuming waiting for the stain to take effect. The sample preparation procedure of our method is relatively simple compared with histochemical and immunological methods. Specimen fixation is still required and we compared specimen fixed in $4 \%$ paraformaldehyde for $24 \mathrm{~h}$ and $95 \%$ ethanol for $20 \mathrm{~min}$. There was no significant difference in DIC imaging results between the two fixation methods.

We showed that the microstructure of the two nerves can be used for identification of the nerve type. Nerve fibers were assumed to bundle together and run in parallel with the nerve trunk. While our result shows that nerve fibers in the two types of nerve may have different running patterns. With nerve sections of a certain thickness, this difference can be obviously shown in the cross-section images of nerve sections. IR-DIC was used to get better imaging result of the thick sample, which also allowed a label-free microscopic imaging.
The microstructure of peripheral nerve has previously been investigated. A number of studies also employed the process of dissecting nerves and evaluated their cross-sectional structure. ${ }^{18,19}$ Parameters such as the diameter of nerve trunk and fascicle, number of fascicles, were evaluated. It was found that branching and plexus formation generally occur between the fascicles in the proximal part of the nerve, and this pattern becomes less marked distally. ${ }^{20}$ However, the relationship between nerve type and the detailed running pattern of the nerve fibers inside the fascicles is still unknown.

Conventionally, staining process is required for the microscopic imaging of peripheral nerves, and thin sections were used in order to get better staining result. We showed that, with thin nerve sections, the distribution pattern of internal nerve fibers cannot be distinguished between motor and sensory nerves. And due to the high scattering properties of peripheral nerves, wide field imaging can only be used for imaging the surface of the sample, and the microstructure inside cannot be identified. We have employed confocal microscope in combination with fluorescent labeling to show the different microstructures of motor and sensory nerves. In this paper, we showed that, if nerve sections with appropriate thickness were used, it is possible to distinguish the two types of nerves with direct IR-DIC imaging of unlabeled nerve sections. The two types can be identified according to their different DIC image patterns.

Our method shows the potential to differentiate between motor and sensory nerves. More detailed work on animal model and human samples can be carried out to explore the difference at more distal locations of the peripheral nerve. The functional implication for the twisted running pattern of nerve fibers inside the ventral nerve root can also be studied.

\section{Acknowledgment}

The research was supported by National Natural Science Foundation of China (Grant Nos. 61475059, 81371968, and 81401791). D. Chen, Y. Wu and T. Sui contributed equally to this work.

\section{References}

1. T. Matsuyama, M. Mackay, R. Midha, "Peripheral nerve repair and grafting techniques: a review," Neurol. Med. Chir. (Tokyo) 40, 187-199 (2000). 
2. J. A. Chow, A. L. Van Beek, D. L. Meyer, M. C. Johnson, "Surgical significance of the motor fascicular group of the ulnar nerve in the forearm," J. Hand Surg. [Am] 10, 867-872 (1985).

3. S. Sunderland, "Anatomical features of nerve trunks in relation to nerve injury and nerve repair," Clin. Neurosurg. 17, 38-62 (1970).

4. S. Sunderland, "Advances in diagnosis and treatment of root and peripheral nerve injury," $A d v$. Neurol. 22, 271-305 (1979).

5. M. Deutinger, "Clinical and electroneurographic evaluation of sensory motor-differentiated nerve repair in the hand," J. Neurosurg. 78, 709-713 (1993).

6. H. Gruber, "Acetylcholinesterase-histochemical differentiation between motor and sensory nerve fibers," Brain Res. 51, 207-214 (1973).

7. X. S. Gu, "Rapid immunostaining of live nerve for identification of sensory and motor fasciculi," Chin. Med. J. (Engl.) 105, 949-952 (1992).

8. Y. Kawasaki, "Identification of myelinated motor and sensory axons in a regenerating mixed nerve," J. Hand Surg. $[\mathrm{Am}]$ 25, 104-111 (2000).

9. W. J. Betz, F. Mao, G. S. Bewick, "Activity-dependent fluorescent staining and destaining of living vertebrate motor nerve terminals," J. Neurosci. 12, 363-375 (1992).

10. K. A. Carson, "Carbonic-anhydrase histochemistry - a potential diagnostic method for peripheralnerve repair," Clin. Plast. Surg. 12, 227-232 (1985).

11. Y. Wu, T. Sui, X. Cao, X. Lv, S. Zeng, P. Sun, "Confocal imaging reveals three-dimensional fine structure difference between ventral and dorsal nerve roots," J. Biomed. Opt. 16, 050502 (2011).

12. N. Pavillon, K. Fujita, N. I. Smith, "Multimodal label-free microscopy," J. Innov. Opt. Heal. Sci. 7, 1330009 (2014).

13. R. Cicchi, F. S. Pavon, "Multimodal nonlinear microscopy: A powerful label-free method for supporting standard diagnostics on biological tissues," J. Innov. Opt. Heal. Sci. 7, 1330008 (2014).

14. M. R. Arnison, K. G. Larkin, C. J. R. Sheppard, N. I. Smith, C. J. Cogswell, "Linear phase imaging using differential interference contrast microscopy," J. Microsc. 214, 7-12 (2004).

15. F. Kagalwala, T. Kanade, "Reconstructing specimens using DIC microscope images," IEEE Trans. Syst. Man Cybern. B Cybern. 33, 728-737 (2003).

16. E. D. Salmon, P. Tran, High-resolution videoenhanced differential interference contrast light microscopy, Digital Microscopy, G. Sluder, D. E. Wolf, Eds., Elsevier Netherlands (2007).

17. H. Dodt, W. Zieglgansberger, "Visualizing unstained neurons in living brain slices by infrared DICvideomicroscopy," Brain Res. 537, 333-336 (1990).

18. H. B. Williams, "The importance of internal anatomy of the peripheral nerves to nerve repair in the forearm and hand," Hand Clin. 2, 689-707 (1986).

19. J. O. Güerrissi, M. F. Gil. Miranda, "Intraneural topography of the extratemporal facial nerve: $\mathrm{Mi}$ crosurgical nerve reconstruction," J. Craniofac. Surg. 18, 578-585 (2007).

20. S. E. Mackinnon, "New directions in peripheral nerve surgery," Ann. Plast. Surg. 22, 257-273 (1989). 